\title{
Cost Effectiveness Analysis of Different Management Strategies between Best Supportive Care and Second-line Chemotherapy for Platinum-resistant or Refractory Ovarian Cancer
}

\author{
Phanida Luealon ${ }^{1 *}$,Nipon Khempech ${ }^{1}$,Apichai Vasuratna ${ }^{1}$,Piya Hanvoravongchai ${ }^{2}$, \\ Piyalamporn Havanond ${ }^{3}$
}

\begin{abstract}
Background: There is no standard treatment for patients with platinum-resistant or refractory epithelial ovarian cancer. Single agent chemotherapies have evidence of more efficacy and less toxicity than combination therapy. Most are very expensive, with appreciable toxicity and minimal survival. Since it is difficult to make comparison between outcomes, economic analysis of single-agent chemotherapy regimens and best supportive care may help to make decisions about an appropriate management for the affected patients. Objective: To evaluate the cost effectiveness of second-line chemotherapy compared with best supportive care for patients with platinum-resistant or refractory epithelial ovarian cancer. Materials and Methods: A Markov model was used to estimate the effectiveness and total costs associated with treatments. The hypothetical patient population comprised women aged 55 with platinum-resistant or refractory epithelial ovarian cancer. Four types of alternative treatment options were evaluated: 1) gemcitabine followed by BSC; 2) pegylated liposomal doxorubicin (PLD) followed by BSC; 3) gemcitabine followed by topotecan; and 4) PLD followed by topotecan. Baseline comparator of alternative treatments was BSC. Time horizon of the analysis was 2 years. Health care provider perspective and $3 \%$ discount rate were used to determine the costs of medical treatment in this study. Quality-adjusted life-years (QALY) were used to measure the treatment effectiveness. Treatment effectiveness data were derived from the literature. Costs were calculated from unit cost treatment of epithelial ovarian cancer patients at various stages of disease in King Chulalongkorn Memorial Hospital (KCMH) in the year 2011. Parameter uncertainty was tested in probabilistic sensitivity analysis by using Monte Carlo simulation. One-way sensitivity analysis was used to explore each variable's impact on the uncertainty of the results. Results: Approximated life expectancy of best supportive care was 0.182 years and its total cost was 26,862 Baht. All four alternative treatments increased life expectancy. Life expectancy of gemcitabine followed by BSC, PLD followed by BSC, gemcitabine followed by topotecan and PLD followed by topotecan was $0.510,0.513,0.566$, and 0.570 years, respectively. The total cost of gemcitabine followed by BSC, PLD followed by BSC, gemcitabine followed by topotecan and PLD followed by topotecan was 113,000, 124,302, 139,788 and 151,135 Baht, respectively. PLD followed by topotecan had the highest expected quality-adjusted life-years but was the most expensive of all the above strategies. The incremental cost-effectiveness ratios (ICER) of gemcitabine followed by BSC, PLD followed by BSC, gemcitabine followed by topotecan and PLD followed by topotecan was 344,643, 385,322, 385,856, and 420,299 Baht, respectively. Conclusions: All of the second-line chemotherapy strategies showed certain benefits due to an increased lifeyear gained compared with best supportive care. Moreover, gemcitabine as second-line chemotherapy followed by best supportive care in progressive disease case was likely to be more effective strategy with less cost from health care provider perspective. Gemcitabine was the most cost-effective treatment among all four alternative treatments. ICER is only an economic factor. Treatment decisions should be based on the patient benefit.
\end{abstract}

Keywords: Cost-effectiveness analysis - chemotherapy - best supportive care - recurrent-platinum ovarian cancer

Asian Pac J Cancer Prev, 17 (2), 799-805

Introduction

Ovarian cancer is one of the most common causes of death from gynecologic cancer in the world. Most cases are diagnosed in advanced stage and there is still no effective screening tool for ovarian cancer. (Carter et al., 1997). Surgery is not only a cornerstone of treatment but also plays an important role for the histologic diagnosis and staging.(Berek et al., 2010). Despite high rate of objective response to surgery and primary chemotherapy, relapse

${ }^{1}$ Department of Obstetrics and Gynecology, ${ }^{2}$ Department of Preventive and Social Medicine, Faculty of Medicine, ${ }^{3}$ College of Public Health Science, Chulalongkorn University, Bangkok, Thailand*For correspondence: minky_008@hotmail.com 


\section{Phanida Luealon et al}

rates remain high. (Berek et al., 2010). Patients who have shorter treatment- free intervals (platinum resistance) or have never been in remission at all (refractory ovarian cancer), have objective response rates of second-line chemotherapy of approximately 10-15\%. The standard regimen as second-line chemotherapy in recurrent ovarian cancer has not been established, especially in the patients with a short progression-free interval from the previous treatment. (Arimoto et al., 2012). Second-line chemotherapy for this group is single agent chemotherapies including liposomal doxorubicin, gemcitabine, topotecan, and etoposide. There is no evidence supporting the use of combination chemotherapy with more survival period than single agent. The aim of treatment after relapse is mainly palliative, to control disease symptoms, maintain quality of life, and extend survival duration. (Tangjitgamol et al., 2004). Best supportive care should be considered and offered to the patients, when the second-line chemotherapy may increase risk of toxicity in patients with a history of previous treatments.

Patients who have good performance status without clinically significant comorbidity may wish to continue treatment. (Doyle et al., 1997). Donovan et al. (2002) evaluated the treatment preferences of women with recurrent ovarian cancer and reported that most patients (86\%) initially prefer subsequent therapy, with $25 \%$ never considering the withdrawal of chemotherapy, even when the expected median survival was $<1$ week. (Donovan et al., 2002).

However, many additional chemotherapies strategy are minimal or have no improvement in survival duration, cause more toxicity and use more substantial resources. Since comparing effectiveness of second-line chemotherapy with best supportive care in this setting is difficult. Cost-effective analysis might be the alternative study to compare treatment strategies. The objective of this study was to evaluate the cost-effectiveness of second-line chemotherapy compared with best supportive care for patients with platinum-resistant or refractory epithelial ovarian

\section{Materials and Methods}

A cost-effectiveness analysis was conducted to evaluate second-line chemotherapy compared with best supportive care for patients with platinum-resistant or refractory epithelial ovarian cancer. Markov model was chosen as the structure for the analysis because of various states of disease, required long-term follow up, i.e. until patient's death, and economic end points. Cost data were calculated from unit cost treatment for epithelial ovarian cancer patients in $\mathrm{KCMH}$ in the year 2011. Only direct medical costs were included as health care provider perspective in this study. A 3\% annual discount rate was used, as recommended by WHO and the guideline of health technology assessment in Thailand. The outcomes measurement used in the model were life-years, qualityadjusted life-years (QALYs). Patient's life expectancy was calculated by a sum of times spent in each health state over the time horizon. Incremental cost-effectiveness ratio (ICER) based on the outcomes were analyzed. The time horizon of the model was 2 years because more than $99 \%$ of the patients in this study were dead and data from most trials found that median life expectancy of these patients were less than 1 year (Fleming et al, 2013). Microsoft office Excel 2013 was used to conduct the analysis. This study had been approved by the ethics committee of the Faculty of Medicine, Chulalongkorn University.

\section{Baseline model and estimates Markov model}

A Markov model consisting of five health states (cancer1, progressive disease1, cancer2, progressive disease2, and death) was generated as shown in Figure 1. The patients with platinum-resistant or refractory epithelial ovarian cancer (cancer 1 state) started with two options; the second-line chemotherapy or best supportive care. Those with no progressive disease remained in the same state for the next cycle. With disease progression (progressive disease 1 state), the patients moved to cancer state 2 or to death state. In cancer state 2 , the patients received third-line chemotherapy or BSC and moved to progressive disease 2 state with disease progression. For each of the states, the patients could move to death state. The Markov model ended with the absorbing status death. The cycle length was set for 1 month. Survival and progression-free survival (PFS) data were derived from the review literature and cost data were based on actual charge and unit cost treatment of epithelial ovarian cancer patients at various stages of disease in KCMH. The study used a hypothetical female patient population of 55 years of age with platinum-resistant or refractory epithelial ovarian cancer. The authors made an assumption about patient population from review medical record of patients with platinum-resistant or refractory epithelial ovarian cancer in $\mathrm{KCMH}$. The four types of treatment strategies analyzed included: 1) gemcitabine followed by BSC 2) pegylated liposomal doxorubicin (PLD) followed by (BSC) 3) gemcitabine followed by topotecan and 4) PLD followed by topotecan. Baseline comparator of treatment strategies was BSC.

BSC was included in the evaluation and treatment of symptomatic problems. All medications such as analgesic drugs, hormonal treatment, and vitamins or other palliative supportive interventions or procedures such as blood transfusions, intravenous hydration, abdominal paracentesis, and thoracocentesis were included. Palliative

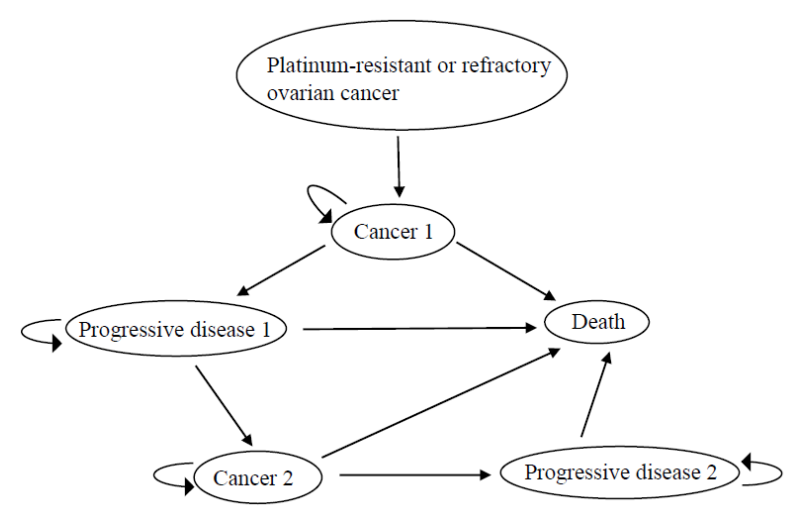

Figure 1. Markov Model of the Platinum-resistant or Refractory Ovarian Cancer 
chemotherapy and herbs were not included to BSC strategy.

In the second-line chemotherapy strategies, gemcitabine at a dose $1,000 \mathrm{mg} / \mathrm{m}^{2}$ was given intravenously on day 1 and days 8 every 3 weeks or PLD at dose $40 \mathrm{mg} / \mathrm{m}^{2}$ was given intravenously on day 1 every 4 weeks until disease progression, thereafter the options of treatment were BSC or third-line chemotherapy.

In the third-line chemotherapy strategy, topotecan at dose $1 \mathrm{mg} / \mathrm{m}^{2}$ was given intravenously for 3 days every 3 weeks until disease progression, then followed by BSC.

\section{Cost inputs}

Cost data were collected from review medical record of patients with platinum-resistant or refractory epithelial ovarian cancer who received gemcitabine (GEMZA, GEMITA), PLD (LIPO-DOX, CALYX), topotecan (HYCAMTIN) and BSC in $\mathrm{KCMH}$ during 2008 to December 2012. Cost of generic and original chemotherapy were included to calculate unit cost, therefore a range of unit cost was showed minimum and maximum value. The cost and price data included in the analysis was the prospective of health care provider as shown in Table 1. The authors used only direct medical costs, consisting of material costs and cost of chemotherapeutic agents, premedication, symptomatic treatment, treatment of toxicity from chemotherapy, and investigation (routine blood works for chemotherapy patients), which occurred in-patient and out-patient department. Capital cost and labor cost were also included. Costs of chemotherapy were calculated for hypothetical 55-year-old woman patients with a body surface area of $1.54 \mathrm{mg} / \mathrm{m} 2$. The unit costs of all treatment strategies were calculated in cost per cycle of 1 month. The authors assumed that the costs per cycle of best supportive care were not different among the four alternatives. Cost data used in the model was shown in Table 1.

\section{Quality of life scores and Utility inputs}

There were very few studies about quality of life in ovarian cancer patients treated with second-line chemotherapy or best supportive care. Srisuttayasathien et al(Srisuttayasathien et al, 2013) found that in the setting of refractory or recurrent epithelial ovarian cancer, patients who received salvage chemotherapy had comparable quality of life scores with patients treated with palliative care alone. The fourth version of Functional Assessment of Cancer therapy-General questionnaire (FACT-G) and general personal questionnaire were used to analyze data of quality of life scores. The median of quality of life score was $76.33(\mathrm{P}=0.334)$. However, the FACT-G scores are an ordinal-level measurement scale and a utility value is quantitative measurement scale, the FACT-G scores were calculated to the EQ-5D utility index according to equation based on previous study. (Srisuttayasathien et al, 2013)

EQ-5D utility index $=0.238+0.014 x G P+0.006 x \mathrm{GE}$ $+0.008 \times \mathrm{GF}$

Where GP is physical well-being; GE is emotional well-being; GF is functional well-being.

Therefore, the patients in both group had the EQ-5D utility index of 0.766 after converted the FACT-G scores to the EQ-5D utility index.

\section{Clinical inputs}

Treatment effectiveness data measured as mean overall survival and progression free survival of best supportive care, second-line and third-line chemotherapy were derived from the review literature.(Mutch et al., 2007; Vergote et al., 2009; Hanker et al., 2012; Nishio et al., 2009) The effectiveness data were calculated to transitional probabilities (P2) of dying during the cycle as shown in equations 1 and 2. (Chaikledkaew, 2009).

$$
\begin{aligned}
\mathrm{R}_{1} & =-\frac{\ln \left(1-\mathrm{P}_{1}\right)}{\mathrm{U}_{1}} \\
\text { and } & \\
\mathrm{P} 2 & =1-\mathrm{e}^{-\mathrm{R} 1 \times \mathrm{U} 2}
\end{aligned}
$$

Where $R_{1}$ is rate per unit of time; $P_{1}$ is probability of overtime period; $\mathrm{U}_{1}$ is overtime period; $\mathrm{P}_{2}$ is probability of unit of time; and $\mathrm{U}_{2}$ is unit of time.

Clinical and utility inputs used in the model were listed in Table 2.

\section{Analysis of uncertainty One-way sensitivity analysis}

The important parameters of model such as treatment efficacy, side effect of chemotherapy, and quality of life scores of treatment and price of chemotherapy were explored in order to analyze parameter uncertainty by one-way sensitivity analysis. The results of one-way sensitivity analysis were based on the result of BSC strategy as comparator.

\section{Probabilistic sensitivity analysis}

Parameters uncertainty was tested in probabilistic sensitivity analysis by using Monte Carlo simulation. The probability distributions for each parameter are shown in Table 1 and 2. Beta distributions were the choice of distribution for probability and utility parameters. Gamma distributions were used for unit cost parameters. Monte Carlo simulation was conducted by using 1,000 trials for the probabilistic analysis. Cost-effectiveness acceptability

\begin{tabular}{|c|c|c|c|c|c|c|}
\hline Variable & $\begin{array}{l}\text { Deterministic } \\
(\mathrm{THB})\end{array}$ & Lower value & Upper value & Distribution & Alpha & Beta \\
\hline Gemcitabine & $11,922.50$ & 6004.16 & 24887.66 & Gamma & 6.125504 & 1946.371 \\
\hline PLD & $54,578.33$ & 52356.91 & 63179.41 & Gamma & 390.8026 & 139.657 \\
\hline Topotecan & $35,950.30$ & 33492.68 & 40748.18 & Gamma & 373.5454 & 96.24077 \\
\hline $\mathrm{BSC}$ & $22,583.20$ & 429.93 & 223137.6 & Gamma & 0.158 & 142926.5 \\
\hline
\end{tabular}

Table 1. Costs Used in the Model

PLD, pegylated liposomal doxorubicin; BSC, best supportive care; Chemotherapy (Patients $55 \mathrm{~kg}, 155 \mathrm{~cm}, \mathrm{BSA} 1.54 \mathrm{mg} / \mathrm{m}^{2}$ ) 
Phanida Luealon et al

curves based on the net benefit framework were provided to illustrate the values of willingness to pay (WTP) for QALY gained.

\section{Results}

Base-case analysis

The estimates of the model inputs and 3\% discount rate being used to determine the results of each strategies, were presented in Table 3. Over the 2 year period of the model, when the patients with platinum-resistant or refractory ovarian cancer received only best supportive care, the overall survival was 2.19 months. The QALYS and total cost of treatment was 0.140 year and 26,862 Baht. However, the second-line chemotherapy, is the other option of treatment because the treatment outcome and total cost were also increased.

In gemcitabine followed by BSC, the overall survival was 6.12 months. The life-year gained and QALYs was 0.510 and 0.390 year, respectively. The incremental cost of treatment was 86,137 Baht. The ICER of treatment was 344,643 Baht/QALY.

In PLD followed by BSC the overall survival was 6.16 months. The life-year gained and QALYs was 0.513 and 0.393 year, respectively. The incremental cost of treatment was 97,440 Baht. The ICER of treatment was 385,322 Baht/QALY.

In gemcitabine followed by topotecan, the overall survival was 6.79 months. The life-year gained and QALYs was 0.566 and 0.432 year, respectively. The incremental cost of treatment was 112,926 Baht. The ICER of treatment was 385,856 Baht/QALY.

In PLD followed by topotecan the overall survival was 6.83 months. The life-year gained and QALYs was 0.570 and 0.435 year, respectively. The incremental cost of treatment was 124,273 Baht. The ICER of treatment was 420,299 Baht/QALY.

BSC was the cheapest treatments option and the lowest life-year gained. Gemcitabine followed by BSC had the lowest incremental cost and the lowest ICER. Although, PLD followed by topotecan also had the highest expected life-year gained and QALYs gained, it had the highest incremental cost and ICER, according to the Table 3.

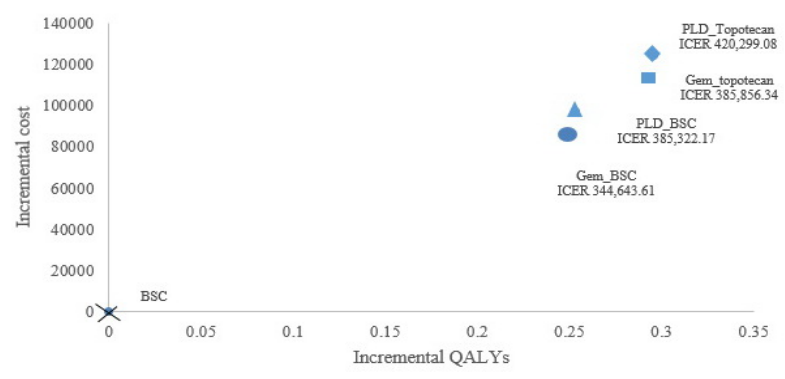

Figure 2. Base-case analysis results (ICERs) of all treatment strategies compared with BSC shown on cost-effectiveness plane. ICERs, incremental costeffectiveness ratios; QALY, quality-adjusted life-year; BSC, best supportive care; PLD, pegylated liposomal doxorubicin

Table 2. Clinical and Utility Inputs used in the Model

\begin{tabular}{|c|c|c|c|c|c|c|c|}
\hline Variable & Deterministic & $\begin{array}{l}\text { Lower } \\
\text { value }\end{array}$ & $\begin{array}{l}\text { Upper } \\
\text { value }\end{array}$ & Distribution & Alpha & Beta & Source data \\
\hline \multicolumn{8}{|l|}{ Survival } \\
\hline $\begin{array}{l}\text { probability of death } \\
\text { gemcitabine }\end{array}$ & 0.053 & 0.04505 & 0.06095 & Beta & 160.6887 & 2889.041 & (Mutch et al, 2007) \\
\hline probability of death PLD & 0.05 & 0.0425 & 0.0575 & Beta & 161.2009 & 3081.817 & (Mutch et al, 2007) \\
\hline $\begin{array}{l}\text { probability of death } \\
\text { topotecan }\end{array}$ & 0.062 & 0.0527 & 0.0713 & Beta & 159.152 & 2422.945 & $\begin{array}{c}\text { (Vergote et al, } \\
\text { 2009) }\end{array}$ \\
\hline probability of death BSC & 0.171 & 0.14535 & 0.19665 & Beta & 140.5416 & 686.1871 & $\begin{array}{l}\text { (Hanker et al, } \\
\text { 2012) }\end{array}$ \\
\hline $\begin{array}{l}\text { probability of death BSC } \\
\left(2^{\text {nd }} \text { relapse }\right)\end{array}$ & 0.156 & 0.1326 & 0.1794 & Beta & 143.1027 & 779.6325 & $\begin{array}{l}\text { (Hanker et al, } \\
\text { 2012) }\end{array}$ \\
\hline $\begin{array}{l}\text { probability of death BSC } \\
\left.\text { ( } 3^{\text {rd }} \text { relapse }\right)\end{array}$ & 0.189 & 0.16065 & 0.21735 & Beta & 137.4683 & 594.1684 & $\begin{array}{c}\text { (Hanker et al, } \\
\text { 2012) }\end{array}$ \\
\hline $\begin{array}{l}\text { probability of PFS } \\
\text { gemcitabine }\end{array}$ & 0.175 & 0.14875 & 0.20125 & Beta & 139.8587 & 664.048 & (Mutch et al, 2007) \\
\hline probability of PFS PLD & 0.2 & 0.17 & 0.23 & Beta & 135.5902 & 546.3609 & (Mutch et al, 2007) \\
\hline $\begin{array}{l}\text { probability of PFS } \\
\text { topotecan }\end{array}$ & 0.149 & 0.12665 & 0.17135 & Beta & 144.2978 & 829.8555 & $\begin{array}{c}\text { (Vergote et al, } \\
\text { 2009) }\end{array}$ \\
\hline $\begin{array}{l}\text { probability continuation } \\
3^{\text {rd }} \text { line chemotherapy } \\
\text { after recurrence } 2^{\text {nd }} \text { line }\end{array}$ & 0.74 & 0.629 & 0.851 & Beta & 43.39182 & 15.59713 & (Villa et al, 1999) \\
\hline \multicolumn{8}{|l|}{ Utilities } \\
\hline Salvage chemotherapy & 0.766 & 0.464 & 0.984 & Beta & 6.799994 & 2.380108 & $\begin{array}{l}\text { (Srisuttayasathien } \\
\text { et al, 2013) }\end{array}$ \\
\hline BSC & 0.766 & 0.464 & 0.984 & Beta & 6.799994 & 2.380108 & $\begin{array}{l}\text { (Srisuttayasathien } \\
\text { et al, 2013) }\end{array}$ \\
\hline
\end{tabular}

PLD, pegylated liposomal doxorubicin; BSC, best supportive care; PFS, progression free survival 
Cost Effectiveness of Best Supportive Care and Second-Line Chemotherapy for Platinum-resistant Ovarian Cancer Table 3. Cost and Effectiveness Results of Treatment Strategies

\begin{tabular}{|c|c|c|c|c|c|}
\hline \multirow[b]{2}{*}{ Outcome } & \multicolumn{3}{|c|}{$2^{\text {nd }}$ line chemotherapy followed by BSC } & \multicolumn{2}{|c|}{$2^{\text {nd }}$ line chemotherapy followed by $3^{\text {rd }}$ line chemotherapy } \\
\hline & BSC & Gemcitabine & PLD & Gemcitabine_topotecan & PLD_topotecan \\
\hline Overall survival (months) & 2.19 & 6.12 & 6.16 & 6.79 & 6.83 \\
\hline Life-year gained & 0.182 & 0.51 & 0.513 & 0.566 & 0.57 \\
\hline QALYs & 0.14 & 0.39 & 0.393 & 0.432 & 0.435 \\
\hline Incremental life-years & - & 0.25 & 0.253 & 0.293 & 0.296 \\
\hline Total cost & $26,862.17$ & $113,000.15$ & $124,302.57$ & $139,788.38$ & $151,135.34$ \\
\hline Incremental cost & - & $86,137.98$ & $97,440.40$ & $112,926.21$ & $124,273.16$ \\
\hline ICER (THB/QALY) & - & $344,643.61$ & $385,322.17$ & $385,856.34$ & $420,299.08$ \\
\hline
\end{tabular}

BSC, best supportive care; PLD, pegylated liposomal doxorubicin; QALYs, Quality-adjusted life-years; ICER, incremental cost-effectiveness ratio

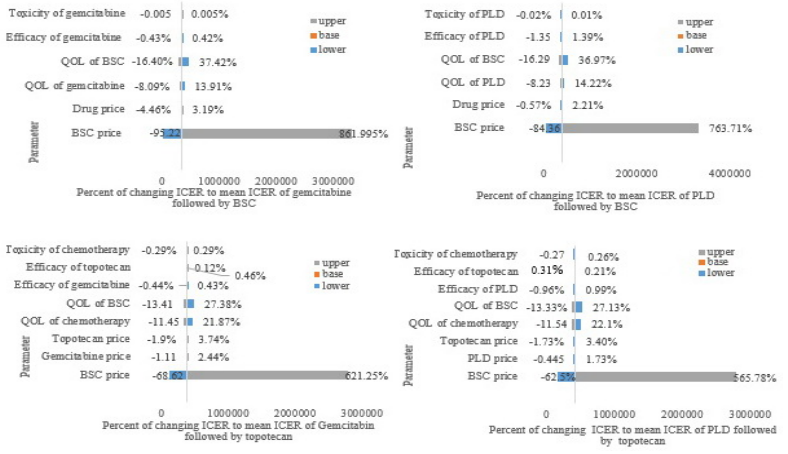

Figure 3. Tornado Diagram Showing the Effects of Parameters on ICER
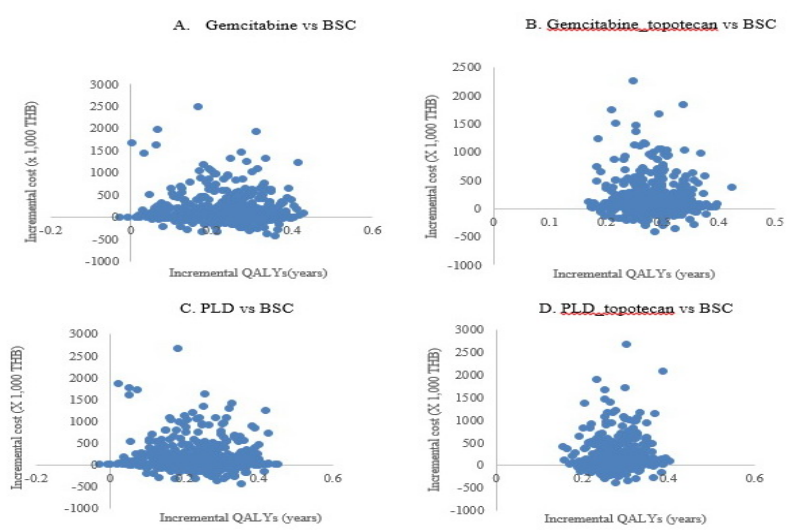

Figure 4. Incremental Cost-effectiveness Comparisons for All Strategies. The scatter plots show the difference in cost and incremental QALYs results of 1000 simulations

While, all treatment strategies increased life year gained and QALYs gained, gemcitabine followed by BSC generated the best result of cost-effectiveness analyses, as shown in Figure 2.

\section{One-way sensitivity analysis}

The influential parameters were selected for the oneway sensitivity analysis, the results were shown in the Tornado diagram (Figure 3 ). The changes of cost of BSC were most sensitive to alter the ICER value in the model, but did not alter the conclusions.

\section{Probabilistic sensitivity analysis}

Figure 4 showed the incremental cost effectiveness scatter plot of 1,000 simulations of each strategy compared to best supportive care. The result showed that all treatment strategies had cost-effectiveness. The cost-effectiveness acceptability curves for the 1,000
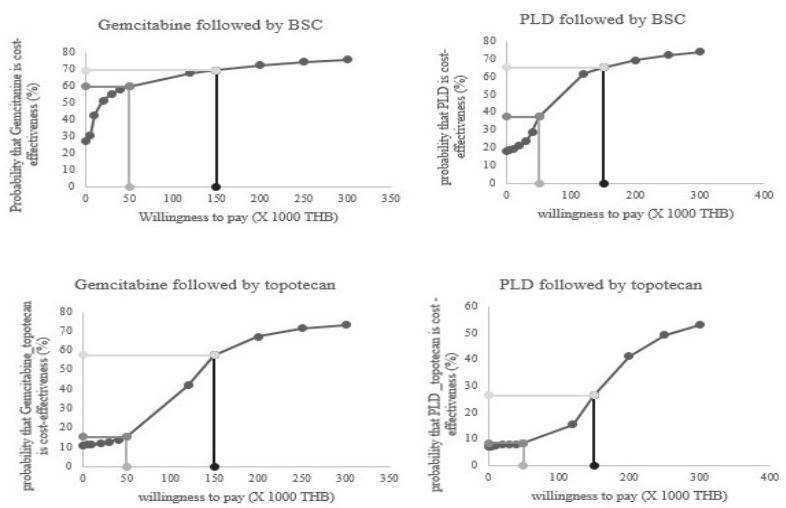

Figure 5. Cost-effectiveness Acceptability Curves

simulations of each strategy were shown in Figure 5. The probability of gemcitabine followed by BSC's incremental cost-effectiveness ratio (ICER) at willing to pay (WTP) thresholds below 50,000 per QALY was $59.7 \%$ of simulations while that of PLD followed by BSC was $37.4 \%$ of simulations. However, WTP thresholds increased below 150,000 per QALY; the probability of both second-line chemotherapy followed by BSC's ICER increased to $69.4 \%$ and $65.3 \%$ of simulations, respectively. If disease progression after second-line chemotherapy at WTP thresholds was below 150,000 per QALY, probability of WTP in BSC strategy being more than probability of WTP in third-line chemotherapy were $11.8 \%$ and $38.8 \%$, respectively. In this study, third-line chemotherapy may have been cost-effective for patients who had previously received gemcitabine for second-line chemotherapy.

\section{Discussion}

In setting of recurrent ovarian cancer with platinumresistant or refractory disease, the patients' response rate to second-line chemotherapy is less effective than the platinum-sensitive disease (Nishio et al, 2009). The expected response rate is similar to or poorer than that of previous treatment and the risk of producing more toxicities in pretreated patients with possibly poor performance status makes some physicians hesitate to treat further (Patnaik et al, 1998). Currently, patients who primarily progress on 2 consecutive chemotherapeutic regimens without evidence of clinical benefit may be offered supportive care, additional therapy, or clinical trials; decisions are made on a highly individual basis. (NCCN, 2012). Therefore, best supportive care is the alternative option and should be considered for these 
patients.

The present study was the first economic evaluation in Thailand to assess the cost-effectiveness of secondline single-agent chemotherapy in recurrent platinumresistant or refractory ovarian cancer compared with best supportive care, using Markov model. The costs of treatment strategies in the models were based on unit cost of treatments of ovarian cancer in $\mathrm{KCMH}$. The present study found that BSC strategy was the least effective, the least expensive one with 0.182-year life-expectancy and 26,862 Baht total cost. The second-line chemotherapy followed by BSC or third-line chemotherapy could extend more patients' life than BSC strategy could (6.12 to 6.83 compared to 2.19 months).

In the transitional states of present models, the assumption of treatment after disease progression was that all patients only received BSC. Therefore, we used the clinical data from the study that the patients were treated with BSC to calculate the transitional probability of state. This explained why the year-life expectancy of the present study was slightly lower than in other studies. (Mutch et al, 2007; Vergote et al, 2009).

In second-line chemotherapy, the total cost of gemcitabine was lower than the total cost of PLD with the approximately same life-year gained. Gemcitabine followed by BSC could be more effective strategy with less cost; ICER was 344,643 Baht compared to BSC strategy's. If the disease progressed after the second-line chemotherapy used, the third-line chemotherapy was still an option to increase life year gained despite the high cost of treatment. However, there was minimal increase in life-year gained in second-line chemotherapy followed by third-line chemotherapy in the present study which was similar to the study of Tangjitgamol et al.(2004).

In the study of Rocconi et al.(2006), it was concluded that only best supportive care (no chemotherapy) was clearly cost-effective while second-line monotherapy was possibly marginal cost-effective (ICER= US\$ 64,104 per year of life saved). The life-year gained from the present study was similar to the previous one, with less total cost of treatment and less ICER. It was probably because of the more expensive cost of medicine of the previous study.

The important parameters such as toxicity, efficacy, and quality of life of chemotherapy, chemotherapy price and cost of treatment of BSC were evaluated for one-way sensitivity analysis. If we increased quality of life, the ICER of treatment would decrease. It was the similar result when we used the QOL scores of Wilailak et al. (2011) to analyze. We would expend more if the cost of toxicity, efficacy, and chemotherapy, and cost of BSC strategy increased. The most influencial factor to this model was the cost of BSC strategy. It may have been because of the difference of treatments, quality of life and survival duration of each patients, so the cost of treatment was in wide range.

For a developing country, World Health Organization (WHO) has recommended medical interventions of which ICER was 3 times below Gross Domestic Product (GPD) per capita income to be deemed cost-effective. (WHO, 2014). In 2011 the GDP per capita for Thailand was 160,556 Baht per year. (Office of the National Economic and Social Development Board 2011. [in Thai]) Therefore the threshold of cost-effectiveness was 481,668 Baht. Though all strategies of this study may be cost-effective, the gemcitabine followed by BSC strategy had the lowest ICER. It was the most cost-effectiveness option.

The strength of the present study was using Markov model to evaluate the cost-effectiveness; there were movement back and forth between points in a model. Probabilistic sensitivity analysis was used to test uncertainty of the model's parameter. The present study used quality of life scores that were collected from the same hospital and converted to the utility value data. It was that the utility data could represent the characteristic of the patients in this study. It may have been due to the similar culture, nationality and life style of the patients and the chemotherapeutic regimen used.

There are some limitations of the present study. The models used health care provider perspective to determine the expense, not including social perspective, however more than $97 \%$ of unit cost was the cost of chemotherapy. There was no change in the unit cost when social perspective cost was included. There have not yet been any well-designed studies on gemcitabine, PLD, and topotecan in Thailand, therefore the present study used the clinical data from review literature. In the study of Suprasert et al. (2012), it was found that response rate of generic gemcitabine in patients with recurrent epithelial ovarian cancer, fallopian tube cancer and primary peritoneal adenocarcinoma was $12.1 \%$ while the median progression free survival was 2 months and median overall survival was 10 months. From the study it could be concluded that generic gemcitabine seemed to have comparable outcomes with original gemcitabine in treating platinum-resistant epithelial ovarian cancer with minimal toxicity especially in patients who received the treatment as a second-line drug after resistance to platinum-based chemotherapy. In this study, there was no direct collection of utility value data.

In conclusion, all of second-line chemotherapy strategies showed certain benefits regarding the increase in life-year gained compared to best supportive care. Gemcitabine as a second-line chemotherapy followed by best supportive care when disease progressed was likely to be more effective strategy with less cost from health care provider perspective. Gemcitabine followed by best supportive care was the most cost-effective treatment among all four alternative treatments. Each additional chemotherapy strategy provided minimal or no improvement in survival period, induced more toxicity and consumed more substantial resources. Further studies are still needed to evaluate the optimal threshold chemotherapy price for Thailand. However, decision to treat should not be based on economic factor alone, a patient's benefit is the most important consideration.

\section{Acknowledgements}

The authors wish to thank the useful comments and suggestions from Associate Professor Nathorn Chaiyakunapruk and Usa Chaikledkaew, PhD. The authors also have a heart-felt gratitude to Sureerat 
Cost Effectiveness of Best Supportive Care and Second-Line Chemotherapy for Platinum-resistant Ovarian Cancer

Ngamkiatphaisan, PhD. for giving the data of cost parameter. The authors, hereby, report no conflicts of interest and no source of financial support to declare.

\section{References}

Carter JR, Chen MD, Fowler JM, et al (1997). The effect of prolonged cycles of chemotherapy on quality of life in gynaecologic cancer patients. J Obstet Gynaecol Res, 23, 197-203

Berek JS, Friedlander M, Hacker NF (2010). Epithelial ovarian, fallopian tube, and peritoneal cancer. In 'Berek \& Hacker's Gynecologic Oncology', Eds Berek J S and Hacker N F. Lippincott Williams \& Wilkins, Philadelphia, 443-508.

Arimoto T, Nakagawa S, Oda K, et al (2012). Second-line chemotherapy with docetaxel and carboplatin in paclitaxel and platinum-pretreated ovarian, fallopian tube, and peritoneal cancer. Med Oncol, 29, 1253-4.

Tangjitgamol S, See HT, Manusirivithaya S, et al (2004). Thirdline chemotherapy in platinum- and paclitaxel-resistant ovarian, fallopian tube, and primary peritoneal carcinoma patients. Int J Gynecol Cancer, 14, 804-14.

Doyle C, Stockler M, Pintilie M, et al (1997). Resource implications of palliative chemotherapy for ovarian cancer. J Clin Oncol, 15, 1000-7.

Donovan KA, Greene PG, Shuster JL, et al (2002). Treatment preferences in recurrent ovarian cancer. Gynecol Oncol, 86, 200-11.

Fleming G, Seidman J, Lengyel E (2013). Epithelial ovarian cancer. in 'principles and practice of gynecologic oncology', Eds Barakat R R, Berchuck A, Markan M, et al. Lippincott Williams \& Wilkins, Philadephia, 757-847

Srisuttayasathien M, Khemapech N (2013). Quality of life in ovarian cancer patients choosing to receive salvage chemotherapy or palliative treatment. Asian Pac J Cancer Prev, 14, 7669-74

Mutch D G, Orlando M, Goss T, et al (2007). Randomized phase III trial of gemcitabine compared with pegylated liposomal doxorubicin in patients with platinum-resistant ovarian cancer. J Clin Oncol, 25, 2811-8

Vergote I, Finkler N, del Campo J, et al (2009). Phase 3 randomised study of canfosfamide (Telcyta, TLK286) versus pegylated liposomal doxorubicin or topotecan as third-line therapy in patients with platinum-refractory or -resistant ovarian cancer. Eur J Cancer, 45, 2324-32.

Hanker L C, Loibl S, Burchardi N, et al (2012). The impact of second to sixth line therapy on survival of relapsed ovarian cancer after primary taxane/platinum-based therapy. Ann Oncol, 23, 2605-12.

Nishio S, Katsumata N, Matsumoto K, et al (2009). Usefulness of third-line chemotherapy for women with recurrent ovarian, fallopian tube, and primary peritoneal cancer who receive platinum/taxane regimens as first-line therapy.J Cancer Res Clin Oncol, 135, 551-7.

Chaikledkaew U (2009). Guideline for estimate life expectancy and transitional probability. (in Thai). In 'Thai National Health Technology Assessment Guidelines', Eds Chaikledkaew U, Teerawatananon Y, Kongpittayachai S, et al. Chulalongkorn University Press, Bangkok, 315-45.

Patnaik A, Doyle C, Oza AM (1998). Palliative therapy in advanced ovarian cancer: balancing patient expectations, quality of life and cost. Anticancer Drugs, 9, 869-78.

NCCN (2012). Epithelial ovarian cancer/fallopian tube cancer/ primary peritoneal cancer.

Rocconi RP, Case AS, Straughn JM, et al (2006). Role of chemotherapy for patients with recurrent platinum-resistant advanced epithelial ovarian cancer: A cost-effectiveness analysis. Cancer, 107, 536-43.

Wilailak S, Lertkhachonsuk AA, Lohacharoenvanich N, et al (2011). Quality of life in gynecologic cancer survivors compared to healthy check-up women. J Gynecol Oncol, 22, 103-9.

World Health Organization (2014). Cost effectiveness and strategic planning (WHO-CHOICE).

Office of the National Economic and Social Development Board (2011. [in Thai]) Bangkok, National Incom of Thailand, Chain Volum Measures: 1990-2010 Edition

Suprasert P, Cheewakriangkrai C, Manopunya M (2012). Outcome of single agent generic gemcitabine in platinumresistant ovarian cancer, fallopian tube cancer and primary peritoneal adenocarcinoma. Asian Pac J Cancer Prev, 13, $517-20$. 